\title{
In-cylinder Temperature Measurements Using Laser Induced Grating Spectroscopy and Two-Colour PLIF
}

\author{
Blane Scott, Christopher Willman, Ben Williams, Paul Ewart and Richard Stone \\ University of Oxford
}

David Richardson

Jaguar Land Rover Limited

\begin{abstract}
In-cylinder temperature measurements are vital for the validation of gasoline engine modelling and useful in their own right for explaining differences in engine performance. The underlying chemical reactions in combustion are highly sensitive to temperature and affect emissions of both NOx and particulate matter. The two techniques described here are complementary, and can be used for insights into the quality of mixture preparation by measurement of the in-cylinder temperature distribution during the compression stroke. The influence of fuel composition on in-cylinder mixture temperatures can also be resolved. Laser Induced Grating Spectroscopy (LIGS) provides point temperature measurements with a pressure dependent precision in the range 0.1 to $1.0 \%$ when the gas composition is well characterized and homogeneous; as the pressure increases the precision improves. The well-established method of Two-Colour Planar Laser-Induced Fluorescence, TC-PLIF allows 2$\mathrm{D}$ temperature distributions to be determined from measurement of fluorescence signals at two different wavelengths. However, the challenge with PLIF is making the qualitative temperature measurements quantitative, so simultaneous LIGS measurements have been used for in situ calibration.
\end{abstract}

\section{Introduction}

Within internal combustion engines, the in-cylinder temperature has a profound effect on many processes including mixture preparation, pollutant formation and combustion performance. The formation of $\mathrm{NO}_{\mathrm{x}}$ and particulate matter is highly sensitive to local in-cylinder temperatures and the rates of chemical reactions that dominate combustion are also highly temperature dependent. In gasoline engines, sites of auto-ignition that can lead to engine knock are dictated by the temperature of the unburned gas during compression [1]. Knock can be particularly damaging in modern, highly boosted Gasoline Direct Injection (GDI) engines, potentially leading to catastrophic failure [2]. For these reasons, the reliable measurement of in-cylinder temperature with high accuracy and precision is of great interest to engine manufacturers, especially for model validation, and in particular when emissions or auto-ignition are being modelled.

Conventional methods for in-cylinder temperature measurements involve the use of fast response thermocouples that can physically affect the measurement environment and provide limited spatial resolution. Therefore, non-intrusive optical and laser-based methods have been developed for in-cylinder gas thermometry. Many of these techniques measure the temperature of a gas at a single 'point' in the combustion chamber. This is usually a small volume of gas in the order of cubic millimetres where two or more laser beams interact. However, 2D or 3D measurements are desirable in order to identify any temperature structure that may be present.

Planar Laser Induced Fluorescence (PLIF) is a well-established laserbased technique that can provide 2-D measurements of in-cylinder temperature, air-fuel distribution and fuel concentration [3]. To quantify PLIF measurements, external calibration is required at known conditions of pressure, temperature and composition. However, these conditions are difficult to replicate under fired conditions, leading to greater measurement uncertainties.

In this work, Laser Induced Grating Spectroscopy (LIGS) has been used for in-cylinder gas thermometry, providing point temperature measurements with high precision. This technique has been shown to achieve measurement uncertainties in the range of 0.1 to $1 \%$ [4]. The precision of the measurements is shown to increase with pressure, which is a clear advantage for engine applications.

In the following sections the LIGS and PLIF techniques are described and their advantages and limitations are discussed. The engines and optical setup used in this work are then described. Preliminary measurements with LIGS demonstrate its ability to resolve small variations in temperature during engine warm-up. Finally, LIGS measurements are used to calibrate 2-D PLIF temperature distributions in situ under motoring and firing conditions for varying air-fuel ratios.

\section{Optical diagnostics for in-cylinder thermometry}

The use of optical diagnostic techniques to measure engine-relevant parameters such as temperature and composition within combustion environments has been thoroughly reviewed [5]-[8]. Two techniques which have seen widespread application to thermometry in internal combustion engines are Planar Laser-Induced Fluorescence, PLIF, and Coherent Anti-Stokes Raman Scattering, CARS.

PLIF thermometry utilises the temperature dependence of either the absorption spectrum for 2-line excitation PLIF [9]-[11], or the fluorescence spectrum for 1-colour [12], [13] or 2-colour detection PLIF [12], [14]-[16] of a molecular tracer such as toluene, acetone or 3-pentanone. In-cylinder thermometry with PLIF has demonstrated precisions of 2-5\% [11], [13], [14], [16] for 2-dimensional measurements with a spatial resolution of the order of $100 \mu \mathrm{m}$. With

Page 1 of 11 
suitable choices of tracer, PLIF thermometry may also be extended to perform simultaneous compositional measurements [3] e.g. for exhaust residuals [17] or fuel/air ratio [10], [18]. Each PLIF technique requires calibration in order to accurately derive temperature from the recorded signals, typically by performing measurements under conditions of known composition, pressure and temperature.

CARS is a four wave mixing technique probing the thermal populations of rotational or vibrational energy levels within a target species. The spectrally resolved signal contains many peaks, whose relative intensities encode information about the Boltzmann population of the levels involved. Fitting a theoretical spectrum to the signal permits the derivation of temperature. For an in-depth review of CARS see reference [19], CARS also features in a review of nonlinear optical techniques applicable to combustion diagnostics [20] .

Vibrational levels of CARS target molecules such as $\mathrm{N}_{2}$ that have large energy gaps have very small thermal populations at temperatures around $300 \mathrm{~K}$. Accordingly, vibrational CARS is typically employed for measurements at elevated temperatures, becoming most sensitive above $1000 \mathrm{~K}$. Single-shot CARS can be performed by replacing the narrow bandwidth Stokes beam, scanned in frequency over time, with a broadband laser. The precision of such measurements is typically limited by noise arising from the modes of the broadband Stokes laser competing for gain, leading to fluctuations in spectral intensity. Use of a modeless broadband laser eliminates this source of noise and can improve the precision of CARS for flame temperature measurements to around $2 \%$ [21]-[23].

At temperatures below $1000 \mathrm{~K}$, the closely spaced rotational energy levels of the ground vibrational state have significant thermal populations. Rotational CARS is therefore suited to performing measurements at temperatures relevant to the in-cylinder conditions of this work, during the compression stroke prior to ignition. Precisions of $3-5 \%$ have been achieved with rotational CARS [24][27] and evaporative cooling effects have been studied [28], [29].

As is the case for both CARS and PLIF measurements, temperatures derived from optical methods which rely on an intensity measurement are susceptible to noise arising from fluctuations in laser pulse energy or spectral intensity, scattered light and other background light sources. In contrast, the LIGS technique derives temperature from the frequency of temporal oscillations in the signal intensity. Noise fluctuations that perturb the intensity of the signal typically have little effect on the derived oscillation period. As such, temperature measurements with LIGS are inherently less sensitive to intensity fluctuations. Combined with the fact that frequencies can be measured with high precision, LIGS temperature measurements can have significantly higher precision than alternative optical methods.

LIGS thermometry has been performed in many combustion environments, including premixed methane/air flames [30], in highpressure sooting flames [31], in combustion engines [4] and in flashboiling sprays [32]. Assuming the conditions of a given environment are known, the accuracy (deviation from the nominally 'correct' value) and precision (variability in the results of repeated measurements under identical conditions) of the LIGS technique may be quantified. Measurements in a static pressure cell under optimal conditions of high pressure (40 bar) and low temperature (300 - 400 $\mathrm{K})$ have demonstrated the potential of LIGS to measure temperatures with both high accuracy $(0.42 \%)$ and high precision $(0.16 \%)$ [33]. The quoted accuracy of $0.42 \%$ was determined by comparison of the mean temperatures derived from 20 individual LIGS signals at each of 9 temperatures across the $300-400 \mathrm{~K}$ range, to the temperatures reported by a two-point calibrated K-type thermocouple mounted in the wall of the cell. The quoted precision of $0.16 \%$ represents the standard deviation of the residuals of the 180 derived temperatures about the corresponding mean temperatures.

The above work uses resonant absorption of the pump beams to produce thermal gratings. By selecting a pump wavelength away from resonance with any absorption features of the gas mixture, electrostrictive gratings can be used to perform LIGS measurements. The theory and experimental application of electrostrictive gratings have been summarized in [34], [35] and the optimal accuracy $(<1 \%)$ and precision $(0.5 \%)$ of electrostrictive LIGS measurements has been evaluated [36]. While the non-resonant nature of electrostrictive gratings permit measurements in air [37], the lower pump pulse intensities required for thermal LIGS and ready availability of absorbing species in a combustion engine environment motivate the use of thermal LIGS in this work and electrostrictive LIGS will not be further discussed.

\section{Laser-Induced Grating Spectroscopy, LIGS}

For a detailed description of the underlying physics of LIGS, the reader is referred to the review of four wave mixing techniques [20] and the two established theoretical descriptions of LIGS grating formation [38]-[41]. To summarise, the LIGS technique can be understood as follows.

LIGS thermometry derives temperature from a measurement of the sound speed in a gas. Absorption of energy from a pair of laser pulses perturbs the local density to form a thermal grating and generates acoustic waves, which modify the density perturbation over time. Directing a probe beam onto the grating and recording the scattered signal allows the evolution of the grating to be monitored and used to derive the local sound speed and therefore temperature.

To form the grating, two short duration laser pulses $(1-10 \mathrm{~ns})$ of wavelength ' $\lambda$ ' are crossed at a small angle, $\theta$, to create an interference pattern of high and low intensity fringes separated by the grating spacing, $\Lambda$,

$$
\Lambda=\frac{\lambda}{2 \sin \left(\frac{\theta}{2}\right)}
$$

The target molecules are electronically excited at the high intensity fringes and deposit this energy, via collisional quenching with the surrounding gas molecules, in the form of heat. This localized heating perturbs the local temperature and pressure, leading to two distinct density perturbations. Firstly, the temperature rise creates a 'thermal grating' with minima of density at the high intensity fringes.

Secondly, the rapid increase in pressure leads to counter-propagating sound waves which sum to form a standing wave. The acoustic oscillations modulate the density perturbation of the thermal grating as the standing wave moves in and out of phase with the stationary thermal grating contribution.

The evolution of the grating can be recorded by scattering a probe beam off the grating onto a detector. The intensity of the scattered signal depends on the magnitude of the periodic density perturbation and therefore is modulated at a frequency, $f_{\text {osc }}$, determined by the local sound speed, $c_{\mathrm{s}}$,

Page 2 of 11 


$$
f_{\text {osc }}=\frac{c_{s}}{\Lambda}
$$

The probe wavelength, $\lambda_{\text {probe}}$, may be freely chosen provided the beam is incident on the grating at the Bragg-scattering angle, $\phi_{B}$, defined by,

$$
\lambda_{\text {probe }}=2 \Lambda \sin \phi_{B}
$$

The scattered signal forms a coherent laser-like beam, aiding in discrimination of the signal from incoherent sources of intensity noise, and follows the temporal evolution of the grating: a singlesided, exponentially decaying signal modulated at a frequency given by ' $f_{\text {osc }}$ ' [Figure 1].

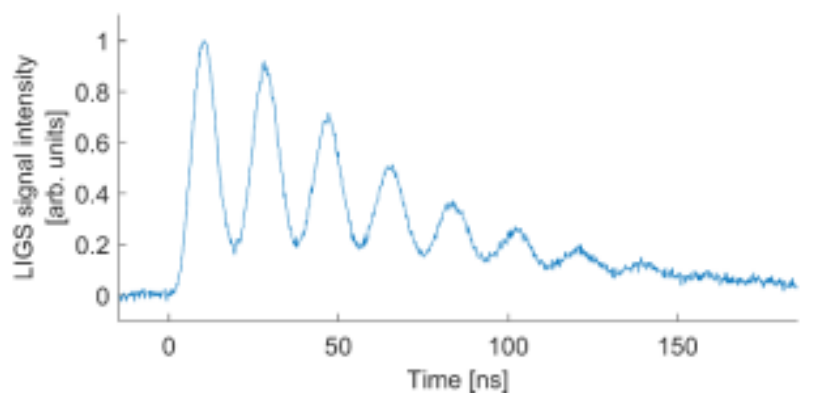

Figure 1 Temporal evolution of a typical single-shot LIGS signal for excitation at time zero, recorded in a static cell at 1.7 bar.

The decay rate of a LIGS signal depends on the diffusion rate of the gas molecules and therefore decreases with increasing pressure. At higher pressures, the longer signals contain a greater number of oscillations, allowing the oscillation frequency to be determined with increased precision.

Measurement of the oscillation frequency allows the sound speed to be determined, given knowledge of the grating spacing, $\Lambda$. The isentropic sound speed is then related to temperature, for the case of an ideal gas, by,

$$
c_{S}=\sqrt{\frac{\gamma k_{B} T}{m}}
$$

where $\gamma$ is the ratio of specific heats at constant pressure to constant volume, $m$ is the mean molecular mass and $k_{\mathrm{B}}$ is Boltzmann's constant. Given knowledge of the local gas composition, $\gamma / m$ can be calculated and temperature can be derived from a measured oscillation frequency,

$$
T=\frac{m \Lambda^{2}}{\gamma k_{B}} f_{o s c}{ }^{2}
$$

As the intensity of the signal does not feature directly in the derivation of temperature, only via measurement of the oscillation frequency, the precision is not directly limited by intensity noise.

The main sources of uncertainty in a LIGS temperature measurement are instead the grating spacing, $\Lambda$, and the local gas composition, $\gamma / m$. Derivation of $\Lambda$ from measurements of $f_{\text {osc }}$ under known temperature and composition is more accurate than geometric calculation of $\Lambda$ and once known, the fixed value of $\Lambda$ can be applied to further measurements.

For in-cylinder measurements, unknown variation in the amount of exhaust residuals, air and fuel at the LIGS measurement region leads to uncertainty in the derived temperature due to the dependence on the mean value of $\gamma / \mathrm{m}$. The derived temperature is relatively insensitive to the exchange of exhaust residuals for a stoichiometric fuel/air mixture [4] as the respective $\gamma / m$ ratios differ by only $6 \%$. The error associated with e.g. assuming zero residuals when the actual value is $10 \%$ would be an error in the derived temperature of $0.6 \%$. In contrast, the $\gamma / \mathrm{m}$ value for a typical 'fuel' molecule for the two-component gasoline surrogate fuel used in this work is a factor of 4.4 smaller than that of air. Uncertainty in the fuel concentration therefore dominates the error in derived temperature. Previous work with the AJ133 engine placed an upper limit of 23\% variability in the fuel concentration and this leads to uncertainty in temperature owing to compositional variations of less than $1.3 \%$ [42].

The derivation of temperature from a LIGS signal relies on the measurement of the frequency of the acoustic oscillations, for which there are two alternative approaches. Firstly, the Fourier transform of the recorded signal may be used to determine the frequency content of the signal. The resulting power spectrum of a LIGS signal contains a peak centred at the frequency of the acoustic oscillation, allowing rapid determination of ' $f_{\text {osc' }}$ '. Secondly, a theoretical LIGS signal may be fitted to the experimental data, determining the oscillation frequency as that which provides the best fit to the data. In this work, both the Fourier transform approach and fitting to a computational model developed previously [33] based on the theory of Paul et al. [40] were used.

\section{Two-Colour Planar Laser-Induced Fluorescence, TC- PLIF}

The populations of rotational and vibrational energy levels within a molecule are temperature-dependent and for certain molecules this leads to a fluorescence spectrum which varies with temperature. In the case of toluene, the fluorescence spectrum shifts to longer wavelengths as temperature is increased. The ratio of fluorescence intensities $S_{1} / S_{2}$ emitted over two different spectral windows, $\Delta \lambda_{1}$ and $\Delta \lambda_{2}$, therefore varies with temperature. This 'signal ratio' from two different wavelength regions (hence Two-Colour PLIF) can be calibrated to provide a measurement of temperature [14], [16], [43][45].

The fluorescence signal, $S$, (photons per pixel) detected during PLIF measurements is given by,

$$
S=\frac{E}{h v} V_{p} n_{t r} \sigma \phi \frac{\Omega}{4 \pi} \eta_{o p t}
$$

where $E$ is laser fluence, $h$ is Planck's constant, $v$ is pump laser frequency, $V_{\mathrm{p}}$ is the excitation volume imaged onto one pixel, $n_{\mathrm{tr}}$ is tracer number density, $\sigma$ is the absorption cross section of the tracer at the pump wavelength, $\phi$ is the fluorescence quantum yield of the tracer, $\Omega$ is the solid angle subtended by the collection optics and $\eta_{\mathrm{opt}}$ is the transmission efficiency of the collection optics [11] [46].

The 'signal ratio' of TC-PLIF is given by,

Page 3 of 11 


$$
\frac{S_{1}}{S_{2}}=C \frac{\phi\left(\Delta \lambda_{1}, T\right)}{\phi\left(\Delta \lambda_{2}, T\right)}
$$

where $\phi$ is the fluorescence quantum yield as a function of wavelengths within a spectral window and temperature $\left(\Delta \lambda_{\mathrm{i}}, T\right)$ and $C$ is a constant. The ratio method removes the requirement to measure several experimental parameters, such as tracer number density and laser fluence, since they are identical for both $S_{1}$ and $S_{2}$ and therefore cancel during calculation of the ratio. This is advantageous for environments where the tracer number density is unknown or varies across the measurement region. This also removes the need for the tracer to co-evaporate with the base fuel, as required when considering fuel/air ratio measurements. The relative merits of this two-colour detection scheme and the detection of the total fluorescence intensity (one colour method) have been evaluated recently by Peterson et al. [12].

Practical application of the TC-PLIF technique involves exciting tracer molecules within a plane using a sheet of laser light and imaging the resulting fluorescence onto separate cameras, using filters to define a different spectral window for each camera. The two images are registered and the ratio of intensities at each pixel, potentially after spatial averaging to reduce noise at the expense of spatial resolution, provides the 'signal ratio'.

The TC-PLIF 'signal ratio' is typically calibrated in terms of temperature to remove the requirement for an accurate model of the fluorescence quantum yield, $\phi$, by preliminary measurements under known conditions over the range of pressures, temperatures and compositions that are likely to occur for a given experiment. Such calibration methods can be very effective where the conditions of the experiment are well defined and match the calibration conditions [47]. Difficulties arise if there is any uncertainty in how the calibration conditions apply to the unknown conditions within the engine cylinder.

This issue is potentially resolved by calibrating the TC-PLIF 'signal ratio' in situ using an independent thermometry technique. In this work, this is achieved by simultaneously measuring the temperature at a well-defined location within the TC-PLIF images, ensuring that the calibration and measurement conditions are identical by definition. The accuracy of the TC-PLIF calibration is therefore reliant on the accuracy of the LIGS temperature measurement.

\section{Apparatus and experimental procedure}

\section{Optical Engine}

Two single-cylinder DISI optical engines have been used for the results presented in this work. The first optical engine, based on a Jaguar Land Rover AJ133 engine (specifications listed in Table 1), was used for the data presented in the "Engine warm-up measurements" and "Temperature measurements over engine cycle" sections. A stainless steel section with four windows of $16 \mathrm{~mm}$ effective aperture at each quadrant was installed between the cylinder head and liner to provide optical access to the combustion chamber. A standard, flat-topped piston blocked the windows after 45 crank angle degrees before top dead centre ( $45^{\circ}$ BTDC), restricting measurements to before this point. For the results shown in the "Temperature measurements over engine cycle" section, a piston with a rectangular slot was manufactured to allow the transmission of the pump and probe beams when the piston was at top dead centre, which allowed measurements to be made for a full cycle. The

Page 4 of 11 'slotted' piston crown was shaped to compensate for the missing volume to maintain the same compression ratio as the standard piston. A LabVIEW ${ }^{\mathrm{TM}}$ program was used to control injection and ignition timings as well as the triggers for the lasers and cameras. A separate LabVIEW ${ }^{\mathrm{TM}}$ program was used for high-speed data acquisition at one sample every crank angle. A Heypac high pressure pump was used to provide fuel at 150 bar and the intake air was thermostatically controlled at $313 \mathrm{~K}$.

\section{Table 1 AJ133 optical engine operating conditions}

\begin{tabular}{|l|l|}
\hline Bore $\times$ Stroke & $89 \times 90.3 \mathrm{~mm}$ \\
\hline Displacement & $562 \mathrm{~cm}^{3}$ \\
\hline Valves per Cylinder & 2 intake, 2 exhaust \\
\hline Compression Ratio & 11.1 \\
\hline Fuel Pressure & $150 \mathrm{bar}$ \\
\hline Injector & Bosch Multi-hole Nozzle \\
\hline Valve Timing $\left(^{\circ}\right.$ ATDC) & $\begin{array}{l}\text { IVO 34, IVC 242, } \\
\text { EVO 475, EVC 5 }\end{array}$ \\
\hline Start of Injection & $280^{\circ}$ BTDC \\
\hline Start of Ignition & $35^{\circ}$ BTDC \\
\hline Engine speed & $1200 \mathrm{rpm}$ \\
\hline Intake air pressure & $0.4 \mathrm{bar}$ \\
\hline
\end{tabular}

For the measurements presented in the "2-D temperature distributions using Two-Colour PLIF and LIGS" section, a second single-cylinder DISI optical engine was used. This engine is based on the new Jaguar Land Rover Ingenium petrol engine and its specifications are listed in Table 2. Like the AJ133 engine, a stainless steel annulus with orthogonal quartz windows was manufactured to allow optical access to the combustion chamber. The cylinder head has a curved recess that allows the use of larger quartz windows along the axis of the engine (effective aperture of $26 \mathrm{~mm}$ ). A standard flat-topped piston was used and so measurements were restricted to $50^{\circ} \mathrm{BTDC}$ in the compression stroke. A Bowditch piston arrangement with a quartz piston crown window and $45^{\circ}$ mirror on a sliding mount provided access for the PLIF laser sheet from below the combustion chamber.

Table 2 Ingenium optical engine operating conditions

\begin{tabular}{|l|l|}
\hline Bore $\times$ Stroke & $85 \times 90.3 \mathrm{~mm}$ \\
\hline Displacement & $512 \mathrm{~cm}^{3}$ \\
\hline Valves per Cylinder & 2 intake, 2 exhaust \\
\hline Compression Ratio & 9.8 \\
\hline Fuel Pressure & $150 \mathrm{bar}$ \\
\hline Injector & $\begin{array}{l}\text { Bosch Multi-hole } \\
\text { Nozzle }\end{array}$ \\
\hline Valve Timing $\left(^{\circ}\right.$ ATDC) & $\begin{array}{l}\text { IVO 36, IVC 243, } \\
\text { EVO 475, EVC 3 }\end{array}$ \\
\hline Start of Injection & $280^{\circ}$ BTDC \\
\hline Start of Ignition & $20^{\circ}$ BTDC \\
\hline Engine speed & $1200 \mathrm{rpm}$ \\
\hline Intake air pressure & $0.55 \mathrm{bar}$ \\
\hline
\end{tabular}

Exhaust gas recirculation was not present on either engine, so the exhaust residuals are dependent on the clearance volume and valve timing. These were very similar for the AJ133 and Ingenium, leading to an estimated exhaust residuals level of $13 \%$ for both engines. 


\section{Optical Layout}

The geometry of the 3 incident laser beams for the LIGS technique is shown schematically in Figure 2 with respect to the optical engine. The grating was formed by the intersection of two $266 \mathrm{~nm}$ pump pulses of 4 ns duration, with a combined energy of 2-4 mJ. A 50:50 beamsplitter was used to separate the output of an Nd:YAG laser (Continuum Minilite II) into two pulses of equal energy. The $4^{\text {th }}$ harmonic output at $266 \mathrm{~nm}$ was selected for excitation of toluene, present in both the two-component and multi-component gasoline fuels used in this work, which strongly absorbs light at this wavelength.

The narrow crossing angle of the pump beams, $2.5^{\circ}$, is specified by the requirement to pass through the $16 \mathrm{~mm}$ clear aperture windows at the top of the cylinder. This results in a LITGS grating with transverse dimensions of $1 \mathrm{~mm}$, the diameter of the pump beams, and a longitudinal length of approximately $10 \mathrm{~mm}$. The $671 \mathrm{~nm}$ continuous-wave probe beam was generated by a $300 \mathrm{~mW}$ diodepumped solid state laser (CNI, Model no MLL-III-300).

The signal beam was directed, after a path length of around $5 \mathrm{~m}$, onto a photomultiplier with a rise time of $<1 \mathrm{~ns}$. A bandpass filter at $671 \mathrm{~nm}$ removes any interference from scattered pump light, while the long path length between the cylinder and photomultiplier acts to reduce the contribution of incoherent scattered light at the probe wavelength. A fast oscilloscope was used to record the detected signal (for the AJ133 optical engine: a Picoscope 6402 having a bandwidth of $250 \mathrm{MHz}$ and sampling rate of $2.5 \mathrm{GS} / \mathrm{s}$. For the Ingenium engine: a LeCroy Waverunner 6100A with a bandwidth of $1 \mathrm{GHz}$ and a sample rate of $5 \mathrm{GS} / \mathrm{s}$ ).

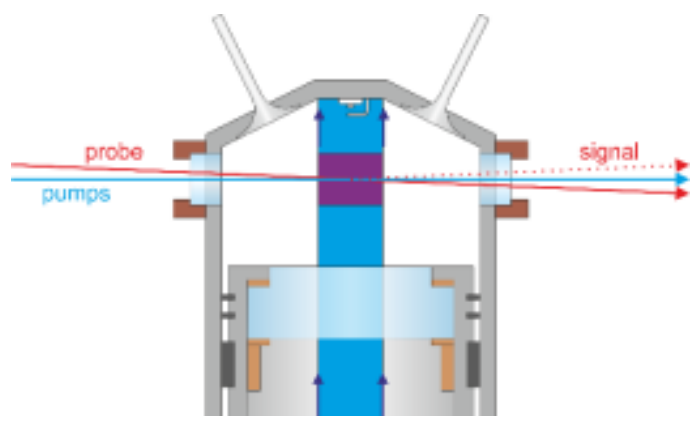

Figure 2 Elevation view of the optical arrangement for application of LIGS and TC-PLIF for in-cylinder measurements. The LIGS beam geometry is detailed for the signal (red dashed), probe (red solid) and pump (blue solid) beams. The excitation sheet for TC-PLIF is directed vertically into the cylinder via a $45^{\circ}$ mirror within the Bowditch piston, entering the cylinder via the piston crown window. The region of the cylinder imaged by the TC-PLIF technique is highlighted by the purple rectangle, with the LIGS intersection point at the centre of this region. The realisation of this is shown in Figure 3.

In addition to the LIGS measurements described above, TC-PLIF measurements were performed simultaneously in the geometry detailed in Figure 2. The excitation sheet at $266 \mathrm{~nm}$ is provided by the $4^{\text {th }}$ harmonic output of a second Nd:YAG laser (Continuum Surelite I-10) with an energy of up to $20 \mathrm{~mJ}$ per pulse. The sheet is directed vertically into the cylinder via a $45^{\circ}$ mirror within the extended Bowditch piston, entering the cylinder through the piston crown window and bisecting the LIGS pump beams. Such an arrangement ensures that the measurement region for LIGS lies within the plane imaged by the TC-PLIF technique as required for insitu calibration [Figure 3].

Page 5 of 11

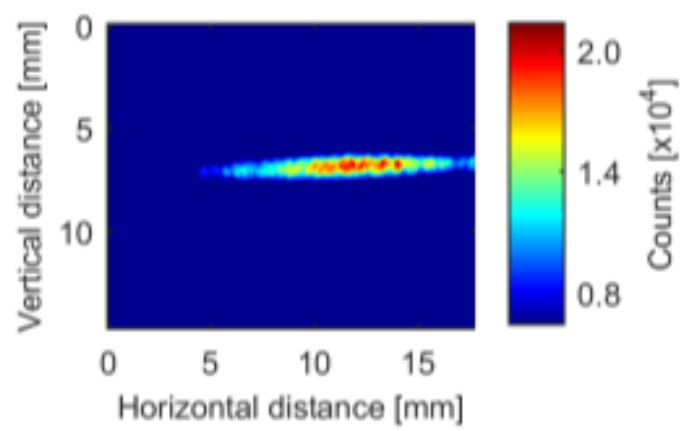

Figure 3 Image of the intersection of the LIGS beams with the TC-PLIF measurement plane [Figure 2], defining the location within the TC-PLIF images to be calibrated using simultaneous LIGS measurements.

After excitation by the incident laser sheet, fluorescence from the toluene component of the fuel is imaged onto two separate intensified CCD (ICCD) cameras [Figure 4]. To define the two spectral windows required for TC-PLIF, a dichroic beamsplitter of edge wavelength $310 \mathrm{~nm}$ (BrightLine-FF310-Di01-25x36) spectrally separates the fluorescence before the ICCD cameras. The long wavelength spectral window is further defined by a $305 \mathrm{~nm}$ longpass filter (BrightLine FF01-300/LP-25), while the short wavelength window, $\Delta \lambda_{2}$, is defined by a $280 \mathrm{~nm}$ bandpass filter (BrightLine -FF01-280/10-25).

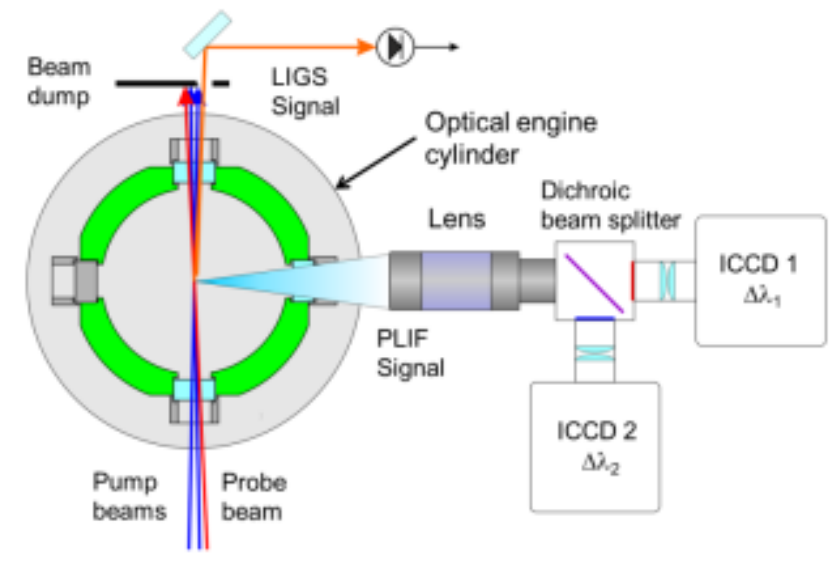

Figure 4 Plan view of the optical engine detailed in Figure 2. LIGS beams intersect the TC-PLIF excitation sheet at the centre of the cylinder. The optical arrangement for the TC-PLIF imaging system consists of an imaging lens and dichroic beamsplitter, followed by two detection arms comprising a pair of relay lenses, either a longpass filter $\left(\Delta \lambda_{1}\right)$ or bandpass filter $\left(\Delta \lambda_{2}\right)$ and an ICCD camera.

Before performing TC-PLIF measurements, the images from both cameras are registered to overlap exactly by recording images of a UV-illuminated grid placed in the plane of the TC-PLIF excitation sheet.

\section{Calibration}

Without knowledge of how the fluorescence quantum yield, $\phi$, varies over the range of conditions within the engine, TC-PLIF alone provides only a qualitative measurement of temperature. An empirical calibration curve, relating the TC-PLIF 'signal ratio' to temperature is generated for each dataset using the simultaneously acquired LIGS measurements, removing the requirement for knowledge of $\phi$ and the calibration constant ' $\mathrm{C}$ ' [Equation 7]. 
For each TC-PLIF image at a given experimental condition, the 'signal ratio' is spatially-averaged over the LIGS measurement region, before averaging this result over multiple cycles to generate a mean 'signal ratio' value. The corresponding mean temperature is calculated by averaging LIGS measurements over the same set of cycles. The ratio of mean temperature to mean 'signal ratio' represents the calibration factor for this condition. Application of this process to the entire dataset generates a calibration curve, which may be applied to the individual spatially-averaged 'signal ratio' values in order to confirm the performance of the calibration [Figure 5]. Application of the calibration curve to the set of TC-PLIF 'signal ratio' images provides quantitative measurement of the in-cylinder temperature distribution.

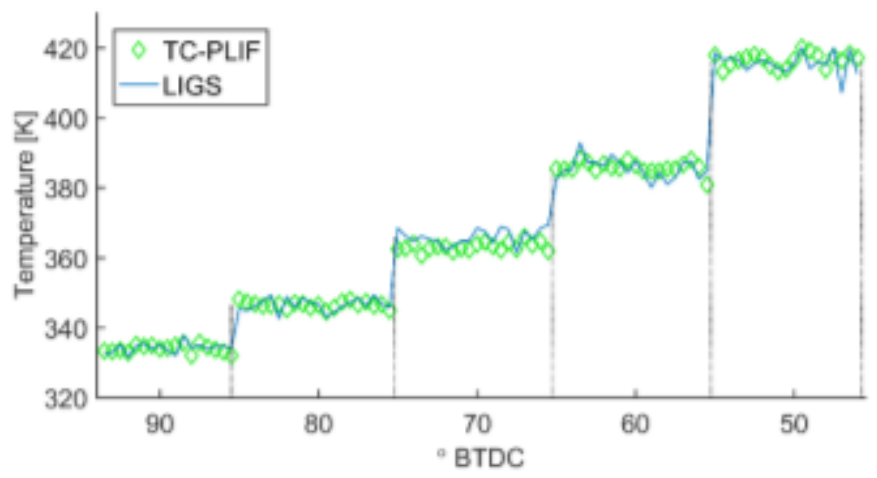

Figure 520 single-shot temperature measurements at each of 5 crank angles during the motored compression stroke using the TC-PLIF and LIGS techniques, illustrating the calibration procedure described in the main text.

\section{Experimental results and analysis}

This section firstly reports the application of LIGS for in-cylinder thermometry, demonstrating the high precision inherent to the LIGS technique. Secondly, preliminary results of TC-PLIF measurements of in-cylinder temperature distributions are presented, having been calibrated in situ with LIGS.

As described above, temperature was derived from the recorded LIGS signals either by extracting the oscillation frequency from the Fourier transform of the LIGS signal, or by fitting a theoretical modelled signal to the experimental data. The relationship between TC-PLIF 'signal ratio' and temperature was calibrated empirically for each dataset in-situ, with reference to the temperatures derived from the simultaneously recorded LIGS signals.

For all measurements reported in this work, toluene was added to a non-fluorescing base fuel to act as the target species for the TC-PLIF and LITGS techniques. A two-component gasoline fuel consisting of a mixture of $70 \%$ iso-octane and $30 \%$ toluene by volume was used for the 'engine warm-up' and 'full cycle' LIGS measurements performed on the AJ133 engine. For the TC-PLIF and LIGS measurements performed on the Ingenium engine a multi-component fuel with volatility similar to gasoline was used (see Table 3).
Table 3 Composition of the multicomponent fuel by volume

\begin{tabular}{|l|c|}
\hline \multicolumn{1}{|c|}{ Species } & Volume fraction \\
\hline Butanes & 0.144 \\
\hline Iso-pentanes & 0.166 \\
\hline Iso-octane & 0.454 \\
\hline n-octane & 0.112 \\
\hline Iso-dodecane & 0.022 \\
\hline n-decanes & 0.101 \\
\hline
\end{tabular}

\section{Engine warm-up measurements}

The high precisions achievable with LIGS enable resolution of small in-cylinder temperature changes of the order of $1 \%$. LIGS signals were recorded for one in every ten cycles at crank angles between $90^{\circ}$ and $50^{\circ}$ BTDC during the initial warm-up phase of the AJ133 optical engine for motored operation at $1200 \mathrm{rpm}$. The air intake temperature and coolant temperature were held at $313 \mathrm{~K} \pm 2 \mathrm{~K}$ and $293 \mathrm{~K} \pm 1 \mathrm{~K}$ respectively. To minimise the potential for compositional uncertainty in the derived temperatures, the two-component fuel was injected into heated air in the plenum, as opposed to the inlet port, with start of injection at $280^{\circ} \mathrm{BTDC}$ in order to provide a homogeneous fuel/air mixture.

Over a period of 10 minutes, four datasets were sequentially recorded whose derived temperatures are shown in Figure 6. The in-cylinder temperature between sequential runs is observed to increase by $3 \mathrm{~K}$ $(0.7 \%)$ at $50^{\circ} \mathrm{BTDC}$.

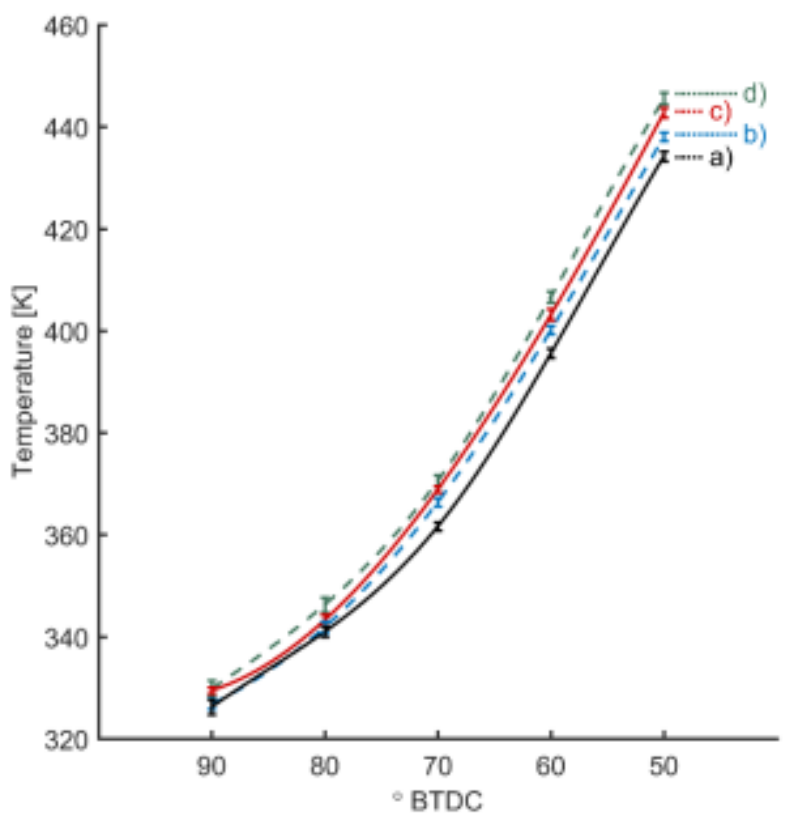

Figure 6 Mean LIGS temperature measurements for sequential datasets a) to d) recorded at 4 minute intervals during the warm-up phase of the engine for motored operation. The error bars represent the standard error of the mean of the 20 measurements at each crank angle. Spline fits to each dataset have been added as guides to the eye.

Page 6 of 11 


\section{Temperature measurements over engine cycle}

The slotted piston provides line-of-sight optical access across the cylinder of the AJ133 optical engine for all crank angles, lifting the restriction of the flat piston which prevents LIGS measurements within $45^{\circ}$ of TDC. Two sets of temperature measurements for motored operation are presented in Figure 7, covering the compression and expansion strokes in $10^{\circ}$ increments.

The improvement in LIGS precision with increasing pressure is demonstrated by the reduction in the error bars towards TDC. By performing measurements in a static engine, the measurement precision at 1 bar (approximately equivalent to the in-cylinder pressure at $90^{\circ} \mathrm{BTDC}$ for the conditions of Figure 7) was found to be $1 \%$. This improves to $0.4 \%$ at $50^{\circ} \mathrm{BTDC}$, approximately 2 bar, as determined by static pressure cell measurements.

Towards the end of the compression stroke, the error bars displaying around $2 \%$ variation in temperature at each crank angle are consistent with the combination of the $0.4 \%$ inherent precision of LIGS and the cyclic in-cylinder peak pressure fluctuations of approximately $1.5 \%$, recorded by a pressure transducer.

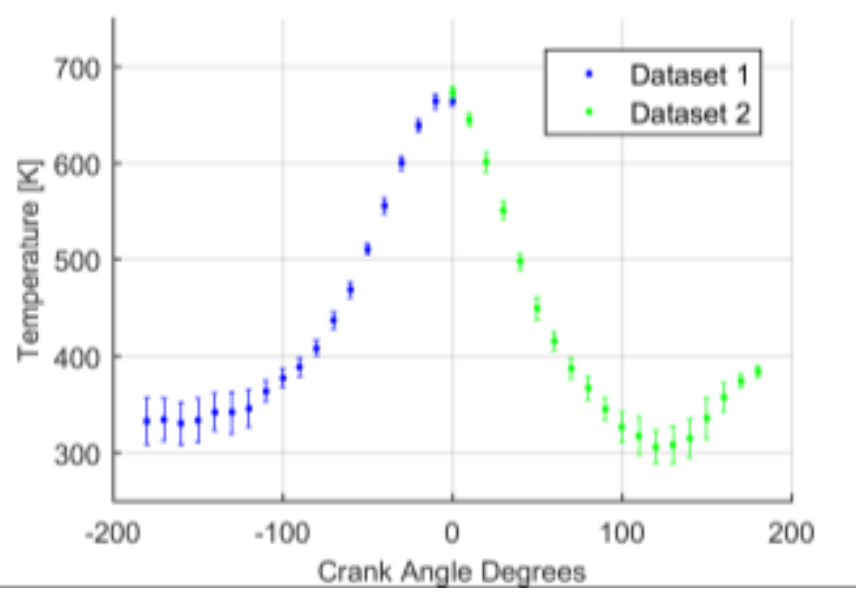

Figure 7 In-cylinder LIGS temperature measurements performed in increments of $10^{\circ}$ throughout the compression stroke and expansion stroke for motored operation. At each crank angle, LIGS temperatures averaged over 50 cycles are displayed, with error bars corresponding to the standard deviation of measured temperatures.

The temperature rise after exhaust valve opening at $115^{\circ}$ ATDC in Figure 7 might at first be unexpected. The inlet valve closure and exhaust valve opening are nearly symmetrical about top centre, and the pressure at IVC is 0.45 bar. During compression and expansion there is leakage of mass and heat, so that when the exhaust valve opens the in-cylinder pressure is lower than at inlet valve closure. The exhaust system is at 1 bar, leading to a reverse flow that compresses the in-cylinder charge. Considering $\gamma$ to be 1.34 for a stoichiometric fuel/air mixture then a pressure ratio of 2.5:1 would result in a temperature rise of $80 \mathrm{~K}$, in reasonable agreement with the observed temperature rise.

\section{2-D temperature distributions using Two-Colour PLIF and LIGS}

LIGS signals and TC-PLIF images were recorded simultaneously during the compression stroke of the Ingenium optical engine at crank angles from $90^{\circ}$ to $50^{\circ} \mathrm{BTDC}$ in increments of $10^{\circ}$. Data was acquired from one in every ten cycles due to the limitation of the ICCD cameras' repetition rate.

The engine was operated under both motored and fired conditions, with direct injection. Air intake temperature was held at $313 \mathrm{~K}$, with a coolant temperature of $358 \mathrm{~K}$. The injection duration for the GDI injector were selected to ensure stoichiometry $(\lambda=1.00 \pm 0.01)$ of the fuel/air mixture using the multi-component gasoline fuel.

Calibration of the TC-PLIF imaging system was performed at $90^{\circ} \mathrm{BTDC}$ using homogeneous distributions of the fuel/air mixture, generated by early injection at $280^{\circ}$ BTDC for motored operation. Temperatures derived from each set of LIGS measurements were used for in-situ calibration of temperature distributions derived from the TC-PLIF images.

\section{Temperature distributions during compression stroke for motored and fired operation}

For motored operation, homogeneous temperature distributions are recorded using TC-PLIF throughout the available range of compression stroke crank angles [Figure 8]. The spatially averaged temperatures show an increase of $86 \mathrm{~K}$ from $339 \pm 11 \mathrm{~K}$ at $90^{\circ} \mathrm{BTDC}$ to $425 \pm 17 \mathrm{~K}$ at $50^{\circ} \mathrm{BTDC}$.

The fluctuations in reported temperature across each TC-PLIF image arise from the low fluorescence signal intensities within the two selected spectral windows and subsequent amplification of the signal at each ICCD camera. Each image has been spatially averaged, resulting in an effective resolution of $0.2 \mathrm{~mm}$, by application of a 2dimensional Gaussian filter with a full-width half-maximum of 7 pixels. The standard deviation of pixel-wise temperatures across the single-shot TC-PLIF images of Figure 8 gives an estimate of the uncertainty in the temperatures reported by TC-PLIF under these motored conditions, varying from $3-4 \%$ between $90^{\circ}$ and $50^{\circ}$ BTDC.

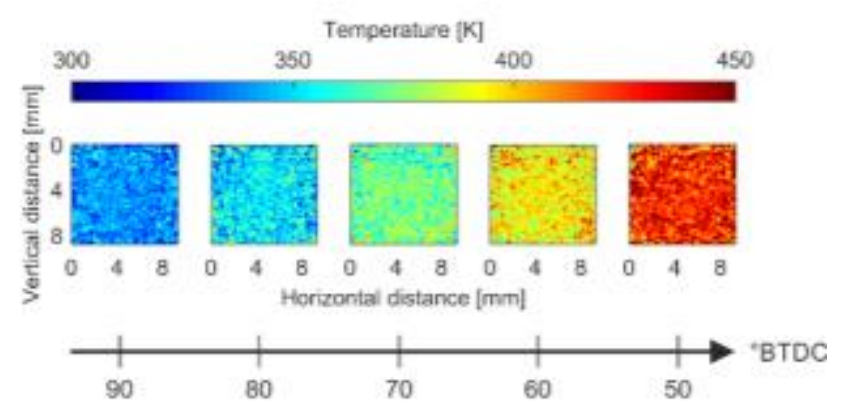

Figure 8 Temperature distributions derived from single-shot TC-PLIF images calibrated in-situ with simultaneous LIGS measurements. Data was recorded at increments of $10^{\circ}$ during the motored compression stroke of the Ingenium optical engine.

Preliminary TC-PLIF measurements for fired operation of the AJ133 optical engine displayed mm-scale inhomogeneity of up to $10 \%$ in the in-cylinder temperature distributions for the range of accessible crank angles, $90^{\circ}$ to $50^{\circ} \mathrm{BTDC}$, during the compression stroke [48].

In contrast, for fired operation of the Ingenium optical engine the TCPLIF temperature distributions are homogeneous during the compression stroke for lean $(\lambda=1.10 \pm 0.04)$, stoichiometric

Page 7 of 11 
$(\lambda=1.00 \pm 0.02)$ and $\operatorname{rich}(\lambda=0.85 \pm 0.03)$ conditions [Figure 9]. The fluctuations in temperature are uniformly distributed across the individual TC-PLIF images and range in magnitude from $2.4 \%$ at $90^{\circ} \mathrm{BTDC}$ to $3.4 \%$ at $50^{\circ} \mathrm{BTDC}$. The Ingenium is a more active combustion system than the AJ133, resulting in increased air motion within the cylinder. This has the potential to produce more homogeneous temperature distributions by improving the mixing of the intake charge with the exhaust gas residuals.

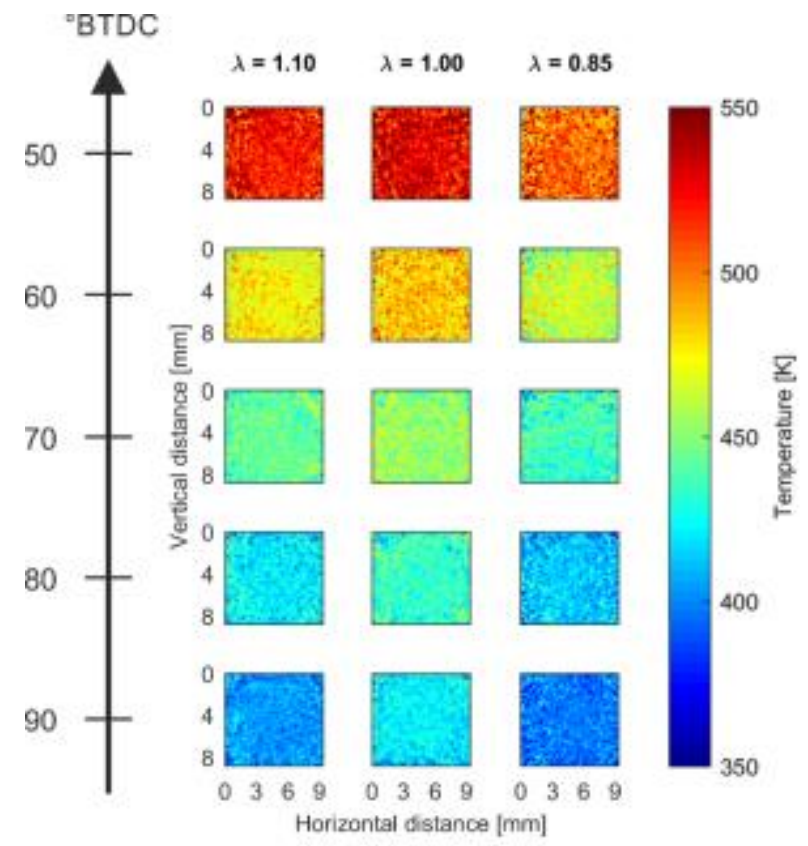

Figure 9 TC-PLIF temperature distributions during the compression stroke for fired operation of the Ingenium optical engine. 1 single-shot image is displayed for each of $5 \mathrm{CAD}$ timings between $90^{\circ}$ and $50^{\circ} \mathrm{BTDC}$, for each of 3 values of relative air/fuel ratio, $\lambda$. Each image covers an $8 \times 9 \mathrm{~mm}$ region in the centre of the cylinder.

The TC-PLIF images presented in Figure 9 illustrate the homogeneity of the in-cylinder temperature distribution over a range of lambda values. However, comparisons of the absolute temperature between datasets of differing lambda may be affected by small differences in engine operation over the course of the two day measurement campaign. Discussion of the influence of lambda on the absolute value of the in-cylinder temperatures is better served by reference to the LIGS data in Figure 10, for which the acquisition rate was increased from $1 \mathrm{~Hz}$ to $10 \mathrm{~Hz}$ in order to minimise firing durations and the potential for physical drifts in temperature between runs.

\section{Effect of varying air-fuel ratio}

By varying the injection duration for a constant air mass flow, LIGS measurements were performed under lean $(\lambda=1.10 \pm 0.04)$, stoichiometric $(\lambda=1.00 \pm 0.02)$ and $\operatorname{rich}(\lambda=0.88 \pm 0.03)$ conditions as measured by a lambda sensor, for both motored and fired operation of the Ingenium optical engine [Figure 10].

For motored operation, relative to the lean $(\lambda=1.10)$ dataset of Figure 10, there is a decrease in temperature of $10 \mathrm{~K}(2.4 \%)$ at $50^{\circ} \mathrm{BTDC}$ for the stoichiometric dataset and $13 \mathrm{~K}(3.2 \%)$ for the rich $(\lambda=0.88)$ dataset. This is consistent with the increase in charge cooling due to the increased mass of fuel injected.
While the intake air and coolant temperatures were held constant, any remaining variation in in-cylinder temperature, as a result of the engine components rising in temperature during engine warm-up, will also have contributed to the measured temperatures of Figure 10. Using data taken under motored stoichiometric conditions before each dataset of Figure 10, the drift in temperature within the cylinder under nominally identical conditions may be estimated. Comparison of the lean and rich datasets with their corresponding stoichiometric reference results in a temperature difference at $50^{\circ} \mathrm{BTDC}$ of $+7 \mathrm{~K}$ $(+1.7 \%)$ for $\lambda=1.10$ and $-9 \mathrm{~K}(-2.1 \%)$ for $\lambda=0.88$.

For fired operation the hot exhaust gas residuals, which remain in the cylinder from the previous fired cycle, will mix with the incoming fresh charge and increase the temperature of the fuel/air/exhaust residuals mixture present during the compression stroke. Also, the fired engine will have higher component temperatures and this too will tend to increase the mixture temperature. This is shown by the $100 \mathrm{~K}$ increase in temperatures measured by LIGS at $50^{\circ} \mathrm{BTDC}$ between motored and fired operation.

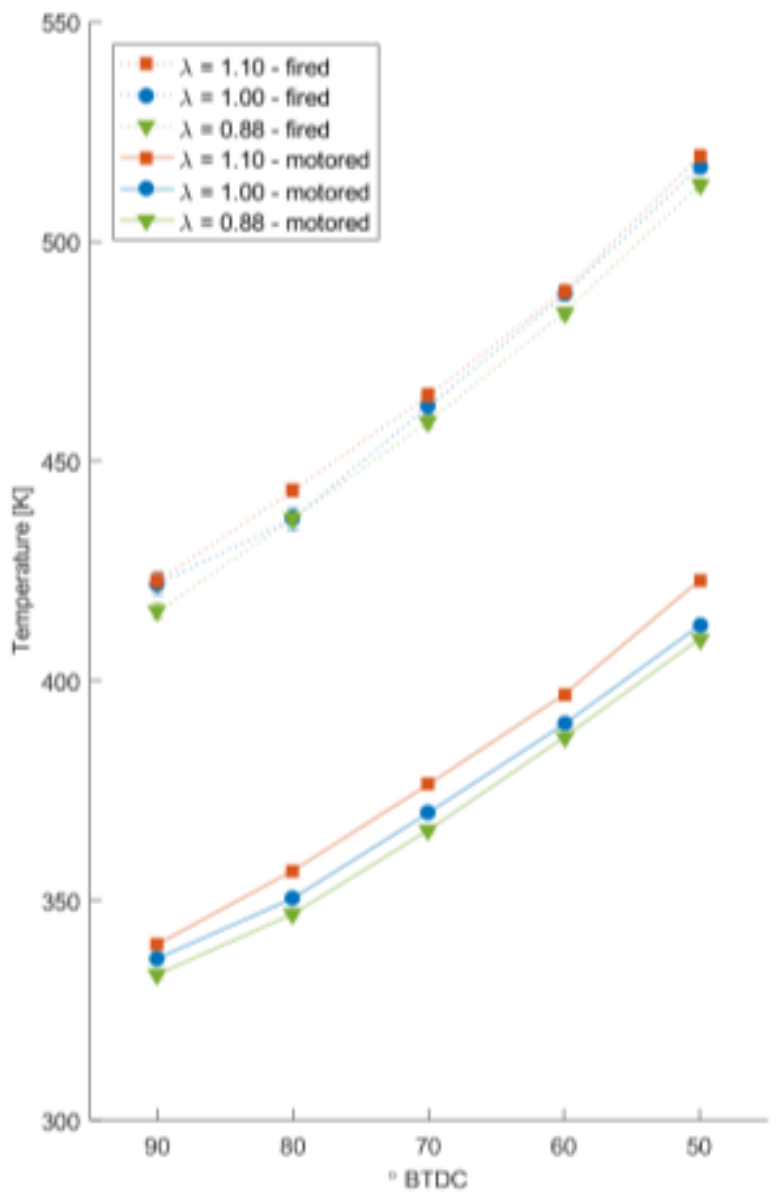

Figure 10 Mean LIGS temperature measurements at increments of $10^{\circ}$ during the compression stroke for motored and fired operation, for each of 3 values of relative air/fuel ratio, $\lambda$. The error bars represent the standard error of the mean of the 50 measurements at each crank angle. Dotted (fired) and solid (motored) lines have been added to each dataset as guides to the eye.

The in-cylinder temperatures for fired operation in Figure 10 are within $0.8 \%$ of the stoichiometric temperature for both lean and rich operation at $50^{\circ}$ BTDC. Peak temperatures produced by combustion of lean mixtures will be lower than those for stoichiometric mixtures,

Page 8 of 11 
however charge cooling will result in a smaller reduction in temperature. Rich mixtures increase the heat capacity of the fuel/air/exhaust residuals mixture, without increasing the heat released by combustion due to the limited supply of oxygen within the mixture. While this suggests rich mixtures will produce cooler incylinder temperatures, in fact the maximum adiabatic flame temperature as a function of $\lambda$ occurs slightly to the rich side of stoichiometry due to dissociation of the products [49]. The temperatures of the rich 'fired' dataset of Figure 10 relative to the stoichiometric case will therefore depend on whether $\lambda=0.88$ produces a higher or lower peak combustion temperature than $\lambda=$ 1.00 , combined with the effect of increased charge cooling of the mixture.

\section{Conclusions}

Simultaneous LIGS and Two-Colour PLIF measurements have been used for high-precision in-cylinder thermometry. Preliminary experiments with LIGS demonstrate that the technique can achieve a precision in the range $0.4-1.0 \%$ for temperature measurements made throughout a full cycle for motored operation. During a 10minute engine warm up period, the LIGS measurements could resolve increases of $\sim 3 \mathrm{~K}$ for sequential runs.

Point temperature measurements with LIGS provided in-situ calibration of in-cylinder temperature distributions imaged with TwoColour PLIF. The effect of varying the air-fuel ratio on the incylinder temperature was investigated. Rich $(\lambda=0.85 \pm 0.03)$, stoichiometric $(\lambda=1.00 \pm 0.02)$ and lean $(\lambda=1.10 \pm 0.04)$ mixtures all showed homogeneous temperature distributions in the compression stroke for both motored and fired operation. This contrasts with similar experiments carried out on the AJ133 engine, in which mm-scale temperature inhomogeneity was observed for fired cycles. This can be explained by the increased in-cylinder air motion of the Ingenium combustion chamber design compared to the AJ133, resulting in better mixing of the exhaust gas residuals with the intake air flow and a more homogeneous temperature distribution. For motored operation, there was a decrease in temperature of $9 \mathrm{~K}$ for the rich condition and an increase of $7 \mathrm{~K}$ for the lean condition as compared to preceding stoichiometric datasets, which demonstrates that differences in evaporative cooling due to variations in the quantity of fuel injected can be resolved with LIGS measurements.

\section{Acknowledgments}

The authors are grateful for support from the Engineering and Physical Science Research Council (EPSRC) UK and from Jaguar Land Rover Ltd.

\section{References}

[1] J. B. Heywood, Internal Combustion Engine Fundamentals. New York: McGraw Hill Inc, 1988.

[2] R. Stone, Introduction to Internal Combustion Engines, 4th ed. Palgrave Macmillan, 2012.

[3] C. Schulz and V. Sick, "Tracer-LIF diagnostics: Quantitative measurement of fuel concentration, temperature and fuel/air ratio in practical combustion systems," Prog. Energy Combust. Sci., vol. 31, no. 1, pp. 75-121, Jan. 2005.
B. Williams, M. Edwards, R. Stone, J. Williams, and P. Ewart, "High precision in-cylinder gas thermometry using Laser Induced Gratings: Quantitative measurement of evaporative cooling with gasoline/alcohol blends in a GDI optical engine," Combust. Flame, vol. 161, no. 1, pp. 270 279, 2014.

A. Eckbreth, Laser Diagnostics for Combustion Temperature and Species, Second Ed. Gordon and Breach Publishers, 1996.

H. Zhao and N. Ladommatos, Engine Combustion Instrumentation and Diagnostics. SAE International, 2001.

J. B. Jeffries and K. Kohse-Höinghaus, Applied Combustion Diagnostics, 1st ed. London: Taylor \& Francis, 2002.

[8] C. Schulz, A. Dreizler, V. Ebert, and J. Wolfrum, "Combustion Diagnostics," in Springer Handbook of Experimental Fluid Mechanics, C. Tropea, A. L. Yarin, and J. F. Foss, Eds. Berlin, Heidelberg: Springer Berlin Heidelberg, 2007, pp. 1241-1315.

[9] M. C. Thurber and R. K. Hanson, "Simultaneous imaging of temperature and mole fraction using acetone planar laserinduced fluorescence," Exp. Fluids, vol. 30, no. 1, pp. 93101, Jan. 2001.

[10] S. Einecke, C. Schulz, and V. Sick, "Measurement of temperature, fuel concentration and equivalence ratio fields using tracer LIF in IC engine combustion," Appl. Phys. B Lasers Opt., vol. 71, no. 5, pp. 717-723, Nov. 2000.

[11] D. A. Rothamer, J. A. Snyder, R. K. Hanson, and R. R. Steeper, "Optimization of a tracer-based PLIF diagnostic for simultaneous imaging of EGR and temperature in IC engines," Appl. Phys. B, vol. 99, no. 1-2, pp. 371-384, Nov. 2009.

[12] B. Peterson, E. Baum, B. Böhm, V. Sick, and A. Dreizler, "Evaluation of toluene LIF thermometry detection strategies applied in an internal combustion engine," Appl. Phys. B, vol. 117, pp. 151-175, 2014.

[13] S. A. Kaiser, M. Schild, and C. Schulz, "Thermal stratification in an internal combustion engine due to wall heat transfer measured by laser-induced fluorescence," Proc. Combust. Inst., Jun. 2012.

M. Luong, R. Zhang, C. Schulz, and V. Sick, "Toluene laser-induced fluorescence for in-cylinder temperature imaging in internal combustion engines," Appl. Phys. B, vol. 91, no. 3-4, pp. 669-675, Apr. 2008.

[15] M. Luong, W. Koban, and C. Schulz, "Novel strategies for imaging temperature distribution using Toluene LIF," $J$. Phys. Conf. Ser., vol. 45, pp. 133-139, Jul. 2006.

[16] B. Peterson, E. Baum, B. Böhm, V. Sick, and A. Dreizler, "High-speed PIV and LIF imaging of temperature stratification in an internal combustion engine," Proc. Combust. Inst., vol. 34, no. 2, pp. 3653-3660, Jul. 2013. 
M. Löffler, F. Beyrau, and A. Leipertz, “Acetone laserinduced fluorescence behavior for the simultaneous quantification of temperature and residual gas distribution in fired spark-ignition engines.," Appl. Opt., vol. 49, no. 1, pp. 37-49, Jan. 2010.

[18] S. Lind, L. Zigan, J. Trost, A. Leipertz, and S. Will, "Simultaneous two-dimensional measurement of fuel-air ratio and temperature in a direct-injection spark-ignition engine using a new tracer-pair laser-induced fluorescence technique," Int. J. Engine Res., vol. 17, no. 1, pp. 120-128, Oct. 2015 .

[19] S. Roy, J. R. Gord, and A. K. Patnaik, "Recent advances in coherent anti-Stokes Raman scattering spectroscopy: Fundamental developments and applications in reacting flows," Prog. Energy Combust. Sci., vol. 36, no. 2, pp. 280306, Apr. 2010.

[20] J. Kiefer and P. Ewart, "Laser diagnostics and minor species detection in combustion using resonant four-wave mixing," Prog. Energy Combust. Sci., vol. 37, no. 5, pp. 525-564, Sep. 2011.

[21] P. Ewart, "A modeless, variable bandwidth, tunable laser," Opt. Commun., vol. 55, no. 2, pp. 124-126, 1985.

[22] P. Snowdon, S. M. Skippon, and P. Ewart, "Rapid communications," Appl. Opt., vol. 30, no. 9, pp. 1008-1010, 1991.

[23] D. R. Snelling, R. A. Sawchuk, and T. Parameswaran, "Noise in single-shot broadband coherent anti-Stokes Raman spectroscopy that employs a modeless dye laser.," Appl. Opt., vol. 33, no. 36, pp. 8295-301, Dec. 1994.

[24] C. Brackmann, J. Bood, M. Afzelius, and P.-E. Bengtsson, "Thermometry in internal combustion engines via dualbroadband rotational coherent anti-Stokes Raman spectroscopy," Meas. Sci. Technol., vol. 15, no. 3, pp. R13$\mathrm{R} 25,2004$

[25] M. C. Weikl, F. Beyrau, and A. Leipertz, "Simultaneous temperature and exhaust-gas recirculation-measurements in a homogeneous charge-compression ignition engine by use of pure rotational coherent anti-Stokes Raman spectroscopy.," Appl. Opt., vol. 45, no. 15, pp. 3646-51, May 2006.

[26] F. Beyrau, A. Bräuer, T. Seeger, and A. Leipertz, "Gasphase temperature measurement in the vaporizing spray of a gasoline direct-injection injector by use of pure rotational coherent anti-Stokes Raman scattering.," Opt. Lett., vol. 29, no. 3, pp. 247-9, Mar. 2004.

[27] T. Seeger and A. Leipertz, "Experimental comparison of single-shot broadband vibrational and dual-broadband pure rotational coherent anti-Stokes Raman scattering in hot air.," Appl. Opt., vol. 35, no. 15, pp. 2665-71, 1996.

[28] S. Hildenbrand et al., "Numerical and experimental study of the vaporization cooling in gasoline direct injection sprays," Proc. Combust. Inst., vol. 31 II, pp. 3067-3073, 2007.
A. Birkigt et al., "Investigation of compression temperature in highly charged spark-ignition engines," Int. J. Engine Res., vol. 12, no. 3, pp. 282-292, 2011. four-wave mixing spectroscopy of $\mathrm{OH}$ in high-pressure flames," Appl. Phys. B Lasers Opt., vol. 67, pp. 667-673, 1998.

[31] M. S. Brown and W. L. Roberts, "Single-Point Thermometry in High-Pressure, Sooting, Premixed Combustion Environments," J. Propuls. Power, vol. 15, no. 1, pp. 119-127, 1999.

B. Roshani et al., "Simultaneous measurements of fuel vapor concentration and temperature in a flash-boiling propane jet using laser-induced gratings," J. Raman Spectrosc., vol. 44, no. 10, pp. 1356-1362, 2013.

[33] R. Stevens and P. Ewart, "Single-shot measurement of temperature and pressure using laser-induced thermal gratings with a long probe pulse," Appl. Phys. B Lasers Opt., vol. 78, no. 1, pp. 111-117, Jan. 2004.

[34] A. Stampanoni-Panariello, D. N. Kozlov, P. P. Radi, and B. Hemmerling, "Gas phase diagnostics by laser-induced gratings I. Theory," Appl. Phys. B Lasers Opt., vol. 81, no. 1, pp. 101-111, 2005.

[35] A. Stampanoni-Panariello, D. N. Kozlov, P. P. Radi, and B. Hemmerling, "Gas-phase diagnostics by laser-induced gratings II. Experiments," Appl. Phys. B Lasers Opt., vol. 81, no. 1, pp. 113-129, 2005 .

[36] S. Schlamp, H. G. Hornung, T. H. Sobota, and E. B. Cummings, "Accuracy and uncertainty of single-shot, nonresonant laser-induced thermal acoustics.," Appl. Opt., vol. 39 , no. 30 , pp. $5477-81,2000$.

R. C. Hart, R. J. Balla, and G. C. Herring, "Nonresonant referenced laser-induced thermal acoustics thermometry in air.," Appl. Opt., vol. 38, no. 3, pp. 577-584, 1999.

[38] E. B. Cummings, "Laser-induced thermal acoustics: simple accurate gas measurements.," Opt. Lett., vol. 19, no. 17, pp. 1361-1363, 1994.

[39] E. B. Cummings, I. A. Leyva, and H. G. Hornung, "Laserinduced thermal acoustics (LITA) signals from finite beams.," Appl. Opt., vol. 34, no. 18, pp. 3290-302, 1995.

[40] P. H. Paul, R. L. Farrow, and P. M. Danehy, "Gas-phase thermal-grating contributions to four-wave mixing," vol. 12, no. 3, pp. 384-392, 1995.

[41] S. Williams, L. A. Rahn, P. H. Paul, J. W. Forsman, and R. N. Zare, "Laser-induced thermal grating effects in flames.," Opt. Lett., vol. 19, no. 21, pp. 1681-1683, 1994.

[42] B. Williams et al., "Quantitative planar laser-induced fluorescence imaging of multi-component fuel/air mixing in a firing gasoline-direct-injection engine: Effects of residual exhaust gas on quantitative PLIF," Combust. Flame, vol.

Page 10 of 11 
157, no. 10, pp. 1866-1878, 2010.

[43] R. Devillers, G. Bruneaux, and C. Schulz, "Investigation of toluene LIF at high pressure and high temperature in an optical engine," Appl. Phys. B Lasers Opt., pp. 735-739, 2009.

[44] P. Lavieille, F. Lemoine, G. Lavergne, and M. Lebouché, "Evaporating and combusting droplet temperature measurements using two-color laser-induced fluorescence," Exp. Fluids, vol. 31, no. 1, pp. 45-55, 2001.

[45] J. Snyder, N. Dronniou, J. Dec, and R. Hanson, "PLIF Measurements of Thermal Stratification in an HCCI Engine under Fired Operation," SAE Int. J. Engines, vol. 4, no. 1, pp. 1669-1688, 2011.

[46] G. Kychakoff, R. D. Howe, and R. K. Hanson, "Quantitative flow visualization technique for measurements in combustion gases.," Appl. Opt., vol. 23, no. 5, pp. 704-712, Mar. 1984.

[47] M. Cundy, P. Trunk, A. Dreizler, and V. Sick, "Gas-phase toluene LIF temperature imaging near surfaces at $10 \mathrm{kHz}$," Exp. Fluids, vol. 51, no. 5, pp. 1169-1176, Jun. 2011.

[48] P. Ewart, C. Willman, B. A. O. Williams, J. Williams, and C. R. Stone, "High precision in-cylinder thermometry using laser induced grating spectroscopy," I Mech E, 2015.

[49] T. Lu, A. Makino, and C. K. Law, "On the OffStoichiometric Peaking of Adiabatic Flame Temperature," vol. 145, pp. 808-819, 2006.

\section{Definitions/Abbreviations}

$\begin{array}{ll}{ }^{\circ} \text { ATDC } & \begin{array}{l}\text { Crank Angle Degrees After Top Dead } \\ \text { Centre }\end{array} \\ { }^{\circ} \text { BTDC } & \begin{array}{l}\text { Crank Angle Degrees Before Top Dead } \\ \text { Centre }\end{array} \\ \text { CARS } & \begin{array}{l}\text { Coherent Anti-Stokes Raman } \\ \text { Spectroscopy }\end{array} \\ \text { DISI } & \text { Direct Injection Spark Ignition } \\ \text { EVC } & \text { Exhaust Valve Closed }\end{array}$

GDI

Gasoline Direct Injection

ICCD

Intensified Charge Coupled Device

$\mathrm{ICE}$

Internal Combustion Engines

IVC

Inlet Valve Closed

IVO

Inlet Valve Open

LIGS

Laser Induced Grating Spectroscopy

TC-PLIF

Two-Colour Planar Laser Induced Fluorescence

TDC

Top Dead Centre

\section{Contact Information}

Richard Stone

Engineering Science, Parks Road, OX1 3PJ

richard.stone@eng.ox.ac.uk 